\title{
Peptide nucleic acid clamping to improve the sensitivity of Ion Torrent-based detection of an oncogenic mutation in KRAS
}

\author{
$\checkmark$ Correspondence \\ martins.Imiguel@gmail.com \\ 8 Disciplines \\ Cancer Biology \\ Q Keywords \\ KRAS \\ Ion Torrent PGM \\ Next-Generation Sequencing \\ Peptide Nucleic Acid Clamp \\ Circulating Tumour DNA \\ it Type of Observation \\ Standalone \\ \& Type of Link \\ Standard Data
}

(- Submitted Apr 25, 2017 (1) Published Jul 21, 2017

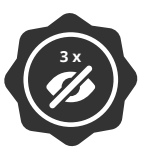

Triple Blind Peer Review The handling editor, the reviewers, and the authors are all blinded during the review process.

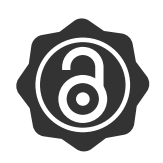

Full Open Access

Supported by the Velux Foundation, the University of Zurich, and the EPFL School of Life Sciences.

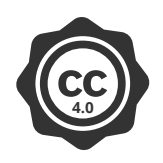

Creative Commons 4.0 This observation is distributed under the terms of the Creative Commons Attribution 4.0 International License.
Callum P Rakhit, Barbara Ottolini, Carolyn Jones, James H Pringle, Jacqueline A Shaw, L. Miguel Martins

CDR Lab, MRC Toxicology Unit; Department of Cancer Studies, University of Leicester; Genomics Facility, MRC Toxicology Unit

\begin{abstract}
Detecting oncogenic changes in the genome of cancer patients is crucial for targeted therapy. Such changes include alterations to KRAS, a GTPase located upstream of several signalling transduction pathways implicated in cancer formation. While nextgeneration sequencing (NGS) allows for comprehensive analysis of a genome, the technology can struggle to detect low frequency variants. To improve the sensitivity of NGS for detecting mutations we created a peptide nucleic acid (PNA) clamp, validated by qPCR, designed to bind wild-type KRAS (WT KRAS) across codon 12 during the PCR amplification stage of a NGS library preparation. We tested the effect of clamping the wild-type $K R A S$ sequence in a reference standard with a $K R A S$ c. $35 \mathrm{G}>\mathrm{A}$ mutation $\left(K R A S^{\mathrm{G} 12 \mathrm{D}}\right)$ at an allelic frequency (AF) of $1.3 \%$ and on circulating-free DNA from a patient harbouring a $K R A S^{\mathrm{G} 12 \mathrm{D}}$ mutation (at an $\mathrm{AF}$ of $3.2 \%$ ). Runs were conducted using $10,5,2.5$ and $1 \mathrm{ng}$ of DNA input. The PNA increased the number of mutant reads and their frequency relative to wild-type calls, allowing for more sensitive detection at all tested concentrations of DNA input.
\end{abstract}

\section{Introduction}

Lung cancer is the leading cause of cancer mortality globally [1]. To better treat this disease, methods to accurately and sensitively detect the somatic mutations driving malignancy are urgently needed. Somatic KRAS mutations are present in $\sim 25-30 \%$ of lung adenocarcinomas [2] [3]. KRAS orchestrates several signalling transduction pathways fundamental to oncogenesis, with alterations in codons 12 and 13 accounting for up to $95 \%$ of all KRAS mutations found in lung adenocarcinoma patients [3], making this hotspot location a key focus of mutation detection assays.

There have been many attempts to inhibit the activity of KRAS (reviewed in [4]) but a recent study described a small molecule inhibitor for $K R A S^{\mathrm{G} 12 \mathrm{C}}$ supporting a novel therapeutic strategy to target this protein [5]. Furthermore, an experimental therapy with trametinib, an inhibitor of MEK1/2, downstream effectors of RAS, combined with docetaxel, may improve the survival of lung cancer patients with KRAS mutations [6]. Hence, patient stratification based on KRAS mutational status may aid the therapeutic response at present, and also as additional RAS-targeted treatments emerge.

Next-generation sequencing (NGS) represents one of the most important technological advances in the molecular diagnosis of cancer in the last 30 years [7]. NGS enables interrogation of multiple regions of the genome for oncogenic changes, on scales unfeasible for more traditional mutation detection methods such as polymerase chain reaction (PCR). However, somatic mutation detection by NGS, using platforms such as the Ion Personal Genome Machine (PGM), is characterised by relatively low sensitivity (down to $1-5 \%$ ) due to its intrinsic sequencing error rates compared to other mutation detection methods [8]. PNA "clamps", first synthesised by Nielsen and colleagues [9], are DNA analogues that inhibit amplification of their target DNA sequences (Fig. 1A). By blocking the amplification of wild-type gene sequences, they became an established tool to aid the detection of oncogenic mutations in several PCR-based methodologies [10]. Here, we tested whether PNA clamps are capable of increasing the detection sensitivity of low frequency oncogenic mutations using the AmpliSeq NGS workflow on the Ion Torrent PGM sequencer. Combining the high-throughput nature of NGS with the sensitivity of PNA clamping, we show, for the selected amplicon, increased detection rates for the low frequency mutation in $K R A S^{\mathrm{G} 12 \mathrm{D}}$. 


\section{Objective}

To test whether the addition of a PNA clamp, which binds WT KRAS at codon 12, can increase the efficacy of mutation detection for KRAS ${ }^{\mathrm{G} 12 \mathrm{D}}$ within a targeted NGS setting.

A

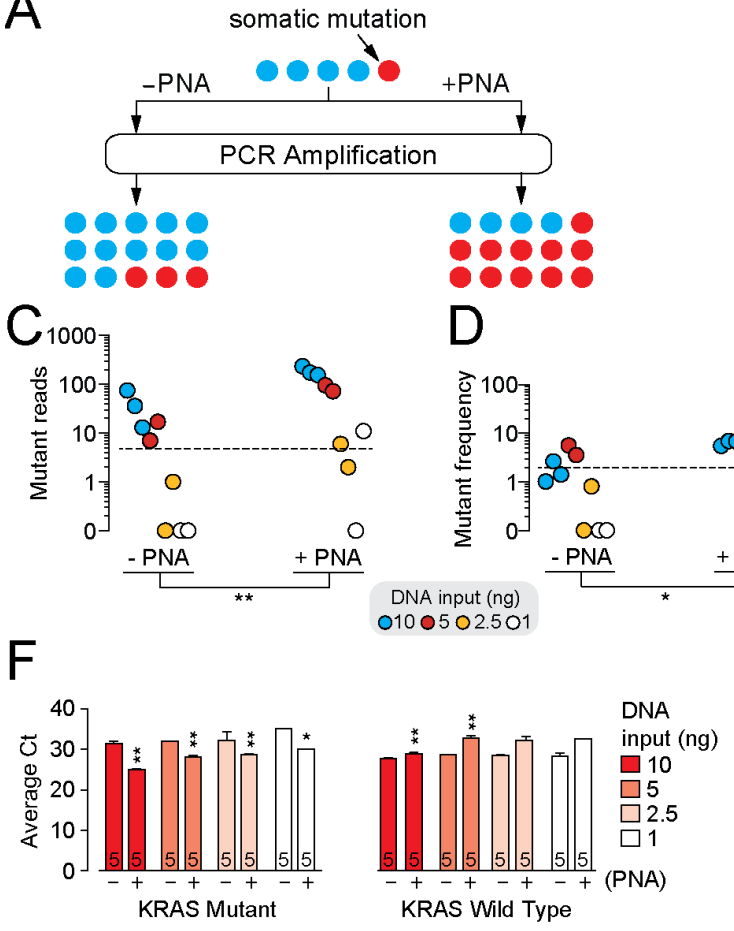

B

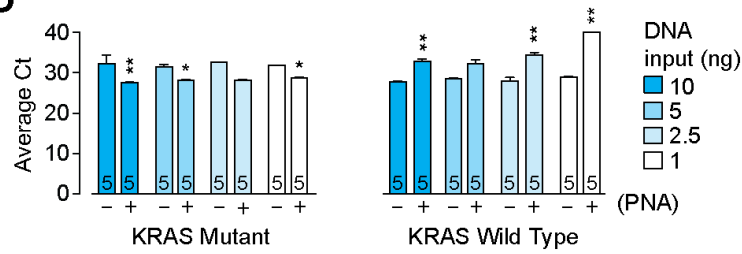

8

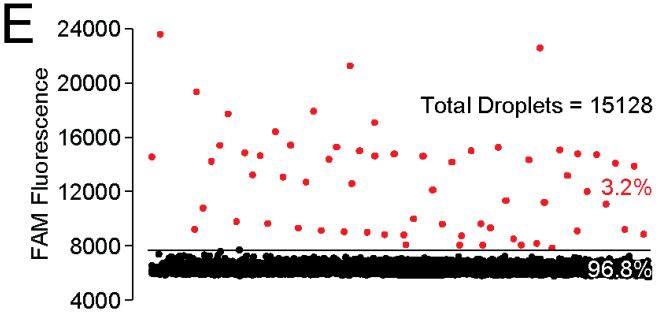

$\mathrm{G}_{1}$

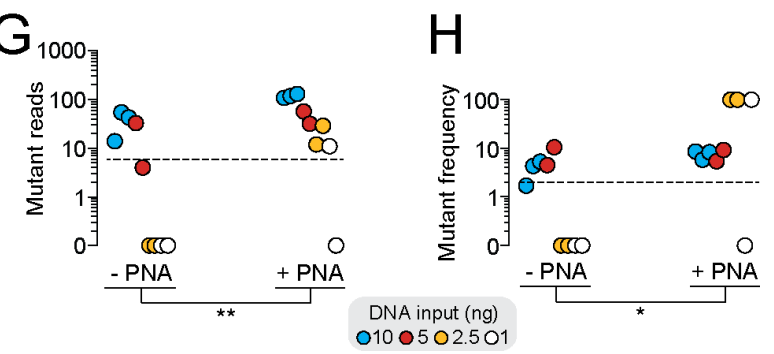

Figure Legend

Figure 1. A PNA clamp for KRAS WT improves the detection of KRAS G12 and G13 mutant codon hotspots by NGS.

(A) Diagram illustrating the effect of PNA clamping on somatic mutation detection by PCR. PNA clamps are designed to bind complimentary DNA sequences: specifically, the PNA binds to WT DNA sequence (blue circles) preventing their amplification during a PCR reaction, and thereby increase the amplification of sequences with a somatic mutation (red circles).

(B) PNA clamp for KRAS WT increases the detection threshold for $K R A S^{\mathrm{G} 12 \mathrm{D}}$ in a DNA reference standard using quantitative PCR (mean \pm S.D., the number of technical replicates (n) is indicated inside the bars).

(C, D) PNA clamp for KRAS WT increases the NGS-detection of $K R A S^{\mathrm{G} 12 \mathrm{D}}$ by determining an increase in read depth for $K R A S^{\mathrm{G} 12 \mathrm{D}}$ calls (C) and in the detected mutant frequency (D). The amount of reference DNA used in each NGS library preparation is indicated. Black dashed lines indicate the lower limit of the detection for the variant considered (VariantCaller v5.0.2.1 "panel optimised" settings).

(E) Detection of a $K R A S^{\mathrm{G} 12 \mathrm{D}}$ in a lung cancer patient by ddPCR. This assay detected a $K R A S^{\mathrm{G} 12 \mathrm{D}}$ mutation present in the patient plasma sample at a frequency of $3.2 \%$ (red dots). To assess the mutant allele fraction, the concentration of mutant DNA in circulating DNA from the plasma was estimated from the Poisson distribution.

(F) PNA clamp for KRAS WT increases the detection threshold for KRAS ${ }^{\mathrm{G} 12 \mathrm{D}}$ in a patient's circulating DNA by qPCR (mean \pm S.D., the number of technical replicates $(\mathrm{n})$ is indicated inside the bars). The amount of DNA template (DNA input) used in the PCR reactions is indicated.

(G, H) PNA clamp for KRAS WT increases the detection sensitivity by NGS of an oncogenic mutation in a clinical cfDNA sample. The PNA increases both the read depth for 
$K R A S^{\mathrm{G} 12 \mathrm{D}}$ calls $(\mathbf{G})$ and the detected mutant frequency $(\mathbf{H})$. The amount of reference DNA used in each NGS library preparation is indicated. Black dashed lines indicate the lower limit of the variant detection threshold. ${ }^{*} p<0.05$, ${ }^{* *} p<0.01$, significant difference between absence and presence of the PNA clamp, Mann-Whitney test.

\section{Peptide Nucleic Acid Clamp}

The PNA clamp utilised was 6 bases in length and designed to clamp the KRAS wild-type sequence only, preventing amplification while leaving mutant sequences fully able to amplify during PCR. The PNA clamp sequence used was CACCAG.

\section{PCR Primers and Probes}

Primers used were those designed for the Ion AmpliSeq ${ }^{\mathrm{TM}}$ Cancer Hotspot Panel v2 sequencing panel (Thermo Fisher Scientific, Paisley, UK). The sequences are as follows: KRAS forward primer 5'CAAAGAATGGTCCTGCACCAGTAATAT-3' and reverse 5'AGGCCTGCTGAAAATGACTGAATATAA-3'. For the genomic region under investigation in KRAS, two TaqMan probes were designed to bind to the same genomic location, with one probe specific to the wild-type sequence, 5'-TTGGAGCTGGTGGCGT-3', and one specific to the G12D mutation, 5'-TGGAGCTGATGGCGT-3' (Thermo Fisher Scientific, Paisley, UK).

\section{Droplet Digital PCR}

All ddPCRs were conducted on a QX20o Droplet Digital PCR System (Bio-Rad, Watford, UK) using the manufacturer's protocol and reagents. Thermal cycling conditions were as follows: $95^{\circ} \mathrm{C}$ for $10 \mathrm{~min}, 40$ cycles of $95^{\circ} \mathrm{C}$ for $15 \mathrm{~s}$ and $60^{\circ} \mathrm{C}$ for $30 \mathrm{~s}, 90^{\circ} \mathrm{C}$ for $10 \mathrm{~min}$. A no template control and a positive control were included in every assay. Analysis was performed according to manufacturer's instructions on QuantaSoft Software (Bio-Rad, Watford, UK).

\section{Quantitative PCR}

Quantitative Real-time PCR was performed on the StepOnePlus Real-Time PCR System (Applied Biosystems, Foster City, CA, USA) using TaqMan Fast Universal PCR Master Mix (Thermo Fisher Scientific, Paisley, UK). Thermal cycling conditions were as follows: $95^{\circ} \mathrm{C}$ for $10 \mathrm{~min}, 40$ cycles of $95^{\circ} \mathrm{C}$ for $15 \mathrm{~s}$ and $60^{\circ} \mathrm{C}$ for $30 \mathrm{~s}$. Reactions were conducted in triplicate, including a no template control and positive control. To account for nondetects in the qPCR data, $C_{t}$ values $>35$ were replaced with a $C_{t}$ of 35 , which has been previously shown to reduce bias [14].

\section{Ion Torrent NGS Protocol}

Library generation followed the protocol described in the Ion AmpliSeq Library Kit 2.0 User Guide using the Ion AmpliSeq Cancer Hotspot Panel v2 primer pool, comprising 207 amplicons covering approximately 2,800 COSMIC mutations from 50 oncogenes and tumor suppressor genes. The relevant PNA clamps were added at a concentration of $10 \mu \mathrm{M}$ per PNA clamp, before the initial multiplex PCR amplification with the primer pool. Two NGS runs were performed on 316v2 chips on an Ion Torrent PGM. These runs consisted of an unmodified run and an NGS run with a KRAS WT PNA clamp. Base calls, PCR duplicate removal and quality control analysis occurred on an Ion Torrent server using tools from the Ion Torrent Suite (v4.o-r76860). Sequencing reads were aligned to the reference human genome 19 (hg 19) using the Torrent Mapping Alignment Program (TMAP). Variant calling was performed using the Torrent Server variantCaller plug-in (v5.0.2.1). The experimental protocols and raw data were deposited in ArrayExpress under accession E-MTAB-5718.

\section{DNA Reference Sample}

The reference sample used was the "Tru-Q 7 (1.3\% Tier) Reference Standard" (Horizon Diagnostics, Cambridge, UK). This pooled DNA contained mutations at a range of frequencies (1.o to $30.0 \%$ ), with the majority of ddPCR-verified mutations having an allelic frequency (AF) of $1.3 \%$.

\section{Patient DNA Sample}

Blood sample collection from an advanced non-small cell lung cancer patient was conducted in accordance with the Declaration of Helsinki. The study protocol was approved by the relevant Ethics Committee. All patients gave written informed consent prior to participation. Blood was taken by venepuncture into K2-EDTA-containing collection tubes (BD Biosciences, San Jose, CA, USA) and processed for cfDNA isolation as de- 
scribed in Page and colleagues [15]. Results \& Discussion

To assess the potential of a PNA clamping to target the WT KRAS codon 12, we first used a quantitative multiplex cell line DNA reference standard (Tru-Q7 reference standard), with an allelic frequency of $K R A S^{\mathrm{G} 12 \mathrm{D}}$ mutation of $1.3 \%$, as determined using droplet digital PCR (ddPCR). Such a frequency would typically be challenging for reliable detection by NGS. We first tested our hypothesis by performing a quantitative PCR analysis (qPCR) of the Tru-Q7 reference DNA in the presence or absence of a PNA clamp designed to bind WT KRAS codon 12 and therefore prevent its amplification. We observed that the addition of the PNA clamp increased the detection threshold for $K R A S^{\mathrm{G}_{12} \mathrm{D}}$ for a range of concentrations of Tru-Q7 reference DNA, with a matched decrease in the detection threshold of the WT KRAS allele (Fig. 1B). Specifically, with 10 ng input template DNA, the PNA shifted the average cycling threshold $\left(\mathrm{C}_{\mathrm{t}}\right)$ for mutant detection from $32.81 \pm 2.05$ to $25.07 \pm 0.21$; with $5 \mathrm{ng}$ input DNA from $34.39 \pm 1.36$ to $28.10 \pm 0.41$; with $2.5 \mathrm{ng}$ input DNA from $32.67 \pm 2.22$ to $28.55 \pm 0.35$ and with $1 \mathrm{ng}$ input DNA from $35 \pm 0$ to $32.00 \pm 0.74$. Next, we tested whether adding this PNA clamp affected the detection of codon 12 mutations by NGS sequencing on the Ion Torrent PGM platform. We observed that both the number of mutant reads and their mutation frequencies relative to wildtype were increased when the PNA clamp was incorporated in the Ion AmpliSeq Cancer Hotspot Panel (v2) workflow (Fig. $1 \mathrm{C}$ and D). We then tested the potential of coupling PNA clamping with NGS in a pre-clinical setting. Circulating-free DNA (cfDNA) is a promising biomarker for a non-invasive estimate of cancer burden amenable to analysis by NGS [11]. We analysed the KRAS status of a patient with advanced non-small cell lung cancer (NCSLC) using plasmatic cfDNA as a source of circulating tumour DNA. We used droplet digital PCR (ddPCR) to detect a $K R A S^{\mathrm{G} 12 \mathrm{D}}$ mutation at an allelic frequency of $3.2 \%$ (Fig. $1 \mathrm{E}$ ).

A qPCR analysis was then performed to assess whether PNA clamping of the WT KRAS allele could improve the detection threshold for this patient oncogenic mutation. Supplementing a range of concentrations of this patient's cfDNA, in presence of PNA clamping, increased the detection threshold for $K R A S^{\mathrm{G} 12 \mathrm{D}}$ by qPCR (Fig. $1 \mathrm{~F}$ ). Specifically, with $10 \mathrm{ng}$ input template DNA, the PNA shifted the average cycling threshold $\left(\mathrm{C}_{\mathrm{t}}\right)$ from 32.71 \pm 2.27 to $27.5 \pm 0.18$; with $5 \mathrm{ng}$ input DNA from $32.80 \pm 2.05$ to $28.15 \pm 0.24$; with 2.5 ng input DNA from $34.51 \pm 1.09$ to $28.15 \pm 0.23$ and with $1 \mathrm{ng}$ input DNA from $34.39 \pm$ 1.16 to $28.64 \pm 0.28$. Next, NGS analysis using a range of concentrations of this patient's cfDNA, in presence of PNA clamping, increased the detection threshold for $K R A S^{\mathrm{G} 12 \mathrm{D}}$, for both the number of mutant reads (Fig. $1 \mathrm{G}$ ) and their mutation frequencies (Fig. $1 \mathrm{H}$ ) relative to wild type. Notably, PNA clamping did not reduce coverage of the targeted amplicon versus all other amplicons and there was no effect on mutant reads or mutant frequency for amplicons not bound by the PNA. We found that the frequency of mutant reads to wild type was increased (Fig. $1 \mathrm{H}$ ) alongside an increase in the total number of mutant reads (Fig. 1I) after addition of the PNA clamp to the sequencing workflow. The use of PNA clamps to improve the sensitivity of mutation detection in NGS has been previously demonstrated for a GNAS mutation [12], clamping a single amplicon in a PCR reaction before DNA sequencing. Here, we have further confirmed the advantages of applying this methodology to a distinct NGS platform, utilising lower concentrations of DNA (10 ng, $5 \mathrm{ng}, 2.5 \mathrm{ng}$ and $1 \mathrm{ng}$ ) that are more amenable for using low DNA yield templates. This finding is of particular relevance to the analysis of cfDNA samples, which are often present at low concentrations and have mutant somatic variants at low frequencies.

Though NGS panels are not used for diagnostic purposes, the establishment of a sensitive sequencing methodology, as the one we outline here, would allow for a broad view of the mutational profile of each patient using only $10 \mathrm{ng}$ of cfDNA. This contrasts with the approaches such as ddPCR. Although being highly sensitive, ddPCR requires $10 \mathrm{ng}$ of cfDNA to assay the status of $K R A S$ codons 12 and 13, without providing information on other oncogenic mutations that might inform targeted cancer therapies.

\section{Conclusions}

Our data show that the addition of a PNA that clamps the wild-type allele for a potential 
somatic mutation in an oncogene such as KRAS increases the detection sensitivity of a specific oncogenic alteration.

\section{Limitations}

The Ion Torrent PGM sequencer has been recently validated as a reliable tool for the quantitative assessment of somatic mutations in KRAS [13]. The addition of a PNA that clamps WT KRAS to this NGS workflow increases the sensitivity for detecting $K R A S^{\mathrm{G} 12 \mathrm{D}}$ mutations, but also changes the nature of the assay from quantitative to qualitative as the true allelic frequency of this specific mutation cannot be determined in presence of PNA clamping.

We would argue that a PNA targeting a specific KRAS amplicon on the Ion Torrent PGM is unlikely to affect the quantitative nature for the remaining 206 amplicons (see Methods section) analysed using this workflow.

Custom sequencing platforms may provide greater opportunities for PNA clamps to increase the sensitivity of a rare event detection. Clamping wild-type sequences within a hotspot region, PNA clamps may suppress the detection of mutations flanking the bound region on a targeted amplicon. To avoid losing resolution on adjacent areas, custom sequencing panels could employ a level of redundancy in amplicon design, generating separate reactions for bound and unbound amplicons for close genomic regions. Nevertheless, this multi-pool primer panel approach needs at least $20 \mathrm{ng}$ of DNA available for multiplex amplification and might not be feasible for samples with limiting concentration as patient-derived plasma cfDNA.

It remains to be determined whether PNA clamps used to increase the detection threshold for a specific mutation by NGS affect the detection threshold for additional mutations in other genomic regions analysed in the multiplexed assay. It also remains to be determined whether several PNAs targeting multiple oncogenic hotspots can be incorporated within the same NGS workflow to increase the detection sensitivity for several oncogenic hotspots.

The use of PNA clamps in NGS represents a method to detect rare events in areas of the genome relevant to patient stratification for targeted therapies, while maintaining the ability to probe broad genomic regions for less common, but potentially informative, somatic alterations, with a multiplexing power that is unavailable to standard PCR-based approaches.

\section{Additional Information}

\section{Methods \\ Peptide Nucleic Acid Clamp}

The PNA clamp utilised was 6 bases in length and designed to clamp the KRAS wild-type sequence only, preventing amplification while leaving mutant sequences fully able to amplify during PCR. The PNA clamp sequence used was CACCAG.

\section{PCR Primers and Probes}

Primers used were those designed for the Ion AmpliSeq ${ }^{\mathrm{TM}}$ Cancer Hotspot Panel v2 sequencing panel (Thermo Fisher Scientific, Paisley, UK). The sequences are as follows: KRAS forward primer 5'CAAAGAATGGTCCTGCACCAGTAATAT-3' and reverse 5'AGGCCTGCTGAAAATGACTGAATATAA-3'. For the genomic region under investigation in KRAS, two TaqMan probes were designed to bind to the same genomic location, with one probe specific to the wild-type sequence, 5'-TTGGAGCTGGTGGCGT-3', and one specific to the G12D mutation, 5'-TGGAGCTGATGGCGT-3' (Thermo Fisher Scientific, Paisley, UK).

\section{Droplet Digital PCR}

All ddPCRs were conducted on a QX20o Droplet Digital PCR System (Bio-Rad, Watford, UK) using the manufacturer's protocol and reagents. Thermal cycling conditions were as follows: $95^{\circ} \mathrm{C}$ for $10 \mathrm{~min}, 40$ cycles of $95^{\circ} \mathrm{C}$ for $15 \mathrm{~s}$ and $60^{\circ} \mathrm{C}$ for $30 \mathrm{~s}, 90^{\circ} \mathrm{C}$ for $10 \mathrm{~min}$. A no template control and a positive control were included in every assay. Analysis was performed according to manufacturer's instructions on QuantaSoft Software (Bio-Rad, Watford, UK). 


\section{Quantitative PCR}

Quantitative Real-time PCR was performed on the StepOnePlus Real-Time PCR System (Applied Biosystems, Foster City, CA, USA) using TaqMan Fast Universal PCR Master Mix (Thermo Fisher Scientific, Paisley, UK). Thermal cycling conditions were as follows: $95^{\circ} \mathrm{C}$ for $10 \mathrm{~min}, 40$ cycles of $95^{\circ} \mathrm{C}$ for $15 \mathrm{~s}$ and $60^{\circ} \mathrm{C}$ for $30 \mathrm{~s}$. Reactions were conducted in triplicate, including a no template control and positive control. To account for nondetects in the qPCR data, $C_{t}$ values $>35$ were replaced with a $C_{t}$ of 35 , which has been previously shown to reduce bias [14].

\section{Ion Torrent NGS Protocol}

Library generation followed the protocol described in the Ion AmpliSeq Library Kit 2.0 User Guide using the Ion AmpliSeq Cancer Hotspot Panel v2 primer pool, comprising 207 amplicons covering approximately 2,800 COSMIC mutations from 50 oncogenes and tumor suppressor genes. The relevant PNA clamps were added at a concentration of $10 \mu \mathrm{M}$ per PNA clamp, before the initial multiplex PCR amplification with the primer pool. Two NGS runs were performed on 316v2 chips on an Ion Torrent PGM. These runs consisted of an unmodified run and an NGS run with a KRAS WT PNA clamp. Base calls, PCR duplicate removal and quality control analysis occurred on an Ion Torrent server using tools from the Ion Torrent Suite (v4.0-r76860). Sequencing reads were aligned to the reference human genome 19 (hg19) using the Torrent Mapping Alignment Program (TMAP). Variant calling was performed using the Torrent Server variantCaller plug-in (v5.o.2.1). The experimental protocols and raw data were deposited in ArrayExpress under accession E-MTAB-5718.

\section{DNA Reference Sample}

The reference sample used was the "Tru-Q 7 (1.3\% Tier) Reference Standard" (Horizon Diagnostics, Cambridge, UK). This pooled DNA contained mutations at a range of frequencies (1.o to $30.0 \%$ ), with the majority of ddPCR-verified mutations having an allelic frequency (AF) of $1.3 \%$.

\section{Patient DNA Sample}

Blood sample collection from an advanced non-small cell lung cancer patient was conducted in accordance with the Declaration of Helsinki. The study protocol was approved by the relevant Ethics Committee. All patients gave written informed consent prior to participation. Blood was taken by venepuncture into K2-EDTA-containing collection tubes (BD Biosciences, San Jose, CA, USA) and processed for cfDNA isolation as described in Page and colleagues [15].

\section{Supplementary Material}

Please see https://sciencematters.io/articles/201706000001.

Funding Statement

This work was funded by the Medical Research Council.

Acknowledgements

We thank the patient for allowing the use of their plasma sample in the study.

Ethics Statement

The study protocol for the cfDNA analysis of cancer patients was approved by the Leicestershire, Rutland and Northamptonshire Research Ethics Committee (University Hospitals of Leicester NHS Trust; REC reference number: 7176).

\section{Citations}

[1] Ferlay Jacques et al. "Estimates of worldwide burden of cancer in 2008: GLOBOCAN 2008". In: International fournal of Cancer 127.12 (June 2010), pp. 2893-2917. DOI:

10.1002/ijc.25516. URL:

https://doi.org/10.1002/ijc. 25516.
[2] Ding Li et al. "Somatic mutations affect key pathways in lung adenocarcinoma”. In: Nature 455.7216 (Oct. 2008), pp. 1069-1075. DOI: $10.1038 /$ nature 07423 . URL: https://doi.org/10.1038/nature07423.

[3] Gregory J. Riely et al. "Frequency and Distinctive Spectrum of KRAS Mutations in Never Smokers with Lung Adenocarcinoma". 
In: Clinical Cancer Research 14.18 (Sept. 2008), pp. 5731-5734. DoI: 10.1158/1078-0432.ccr-08-0646. URL: https : //doi.org/10.1158/1078-0432.ccr-08-0646.

[4] Ostrem Jonathan M. L. and Shokat Kevan M. "Direct small-molecule inhibitors of KRAS: from structural insights to mechanism-based design". In: Nature Reviews Drug Discovery 15.11 (2016), pp. 771-785. DoI: $10.1038 / \mathrm{nrd} .2016 .139$. URL: https://doi.org/10.1038/nrd.2016.139.

[5] Matthew P. Patricelli et al. "Selective Inhibition of Oncogenic KRAS Output with Small Molecules Targeting the Inactive State". In: Cancer Discovery 6.3 (2016), pp. 316-329. DoI: 10.1158/2159-8290.cd-15-1105. uRL: https : //doi.org/10.1158/2159-8290.cd-15-1105.

[6] Gandara David R. et al. "A Phase 1/1b Study Evaluating Trametinib Plus Docetaxel or Pemetrexed in Patients With Advanced Non-Small Cell Lung Cancer". In: Fournal of Thoracic Oncology 12.3 (2017), pp. 556-566. DOI:

10.1016/j.jtho.2016.11.2218. uRL: https : //doi.org/10.1016/j.jtho.2016.11.2218.

[7] Cliff Meldrum, Maria A Doyle, and Richard W Tothill. "Next-Generation Sequencing for Cancer Diagnostics: a Practical Perspective”. In: The Clinical Biochemist Reviews 32 (2011), pp. $177^{-195}$

[8] Quail Michael et al. "A tale of three next generation sequencing platforms: comparison of Ion torrent, pacific biosciences and illumina MiSeq sequencers". In: BMC Genomics 13.1 (2012), p. 341. DOI: $10.1186 / 1471-2164-13-341$. URL: https://doi.org/10.1186/1471-2164-13-341.

[9] P E Nielsen et al. "Sequence-selective recognition of DNA by strand displacement with a thymine-substituted polyamide". In: Science 254.5037 (Dec. 1991), pp. 1497-1500. DOI: $10.1126 /$ science. 1962210 . uRL: https://doi.org/10.1126/science.1962210.
[10] Sholl Lynette M. et al. "Multi-institutional Oncogenic Driver Mutation Analysis in Lung Adenocarcinoma: The Lung Cancer Mutation Consortium Experience". In: fournal of Thoracic Oncology 10.5 (May 2015), pp. 768-777. Dor: $10.1097 /$ jto. 0000000000000516 . URL: https : //doi.org/10.1097/jto.0000000000000516.

[11] Rebecca J. Leary et al. "Development of Personalized Tumor Biomarkers Using Massively Parallel Sequencing”. In: Science Translational Medicine 2.20 (Feb. 2010), 20ra14. DOI: 10.1126/scitranslmed. 3000702 . uRL: https : //doi.org/10.1126/scitranslmed.3000702.

[12] Narumi Satoshi et al. "Quantitative and Sensitive Detection of GNAS Mutations Causing McCune-Albright Syndrome with Next Generation Sequencing”. In: PLOS ONE 8.3 (Mar. 2013), e60525. DoI: $10.1371 /$ journal pone. 0060525 . uRL: https : //doi.org/10.1371/journal.pone.0060525.

[13] Jie Gao et al. "Validation of targeted next-generation sequencing for RAS mutation detection in FFPE colorectal cancer tissues: comparison with Sanger sequencing and ARMS-Scorpion real-time PCR”. In: BMF Open 6.1 (Jan. 2016), eoo9532. Dor: 10.1136/bmjopen-2015-009532. uRL: https : //doi.org/10.1136/bmjopen-2015-009532.

[14] Matthew N. McCall et al. "On non-detects in qPCR data". In: Bioinformatics 30.16 (Apr. 2014), pp. 2310-2316. DOI: 10.1093/bioinformatics/btu239. uRL: https : //doi.org/10.1093/bioinformatics/btu239.

[15] Page Karen et al. "Influence of Plasma Processing on Recovery and Analysis of Circulating Nucleic Acids". In: PLOS ONE 8.10 (Oct. 2013), e77963. DoI:

10.1371/journal pone. 0077963 . uRL: https : //doi.org/10.1371/journal.pone. 0077963. 